\title{
Introduction to the Special Issue on Recent Advances in RF Heating of Plasmas
}

\author{
J. P. Anderson ${ }^{1} \cdot$ C. Darbos ${ }^{2}$
}

Received: 28 October 2015 / Accepted: 29 October 2015 /

Published online: 28 November 2015

(C) Springer Science+Business Media New York 2015

The international experimental reactor ITER (Nuclear Facility INB-174) near Cadarache in France is currently under construction, a ground-breaking project that will bring the world to the verge of sustainable positive net energy gain from fusion. Such a massive undertaking, which includes contributions from Europe, China, India, Japan, Korea, Russia, and the USA, brings with it massive challenges. To create fusion conditions for its magnetically confined plasma, the ITER tokamak will require heating and current drive (H\&CD) systems, a combination of neutral beams (NBs) and radio frequency (RF) H\&CD systems operating at the electron cyclotron (EC) and ion cyclotron (IC) resonances. The EC system is designed to couple a sustained $20 \mathrm{MW}$ to the plasma at a frequency of $170 \mathrm{GHz}$. To achieve this formidable goal, the EC system will have 24 gyrotron oscillators as its driving source, each one capable of generating $1 \mathrm{MW}$. A series of highly efficient low-loss transmission lines consisting of overmoded corrugated waveguide and steerable launcher reflectors will be used to deliver the gyrotrons' power to the tokamak. The IC system will provide another $20 \mathrm{MW}$ of power to the plasma at $50 \mathrm{MHz}$, using large pressurized coaxial lines, each capable of transmitting up to $6 \mathrm{MW}$. This system will have its own complex matching network to minimize power transfer losses. With such power levels, sustaining stable conditions for plasma heating becomes an interesting engineering endeavor. Heating of RF components leads to thermal expansion, mechanical stresses, and changing material properties, all of which must be mitigated while avoiding electrical breakdown caused by high fields.

The annual Workshop on RF Heating Technology of Fusion Plasmas, a joint meeting between experts in the USA, Japan, and Europe, has proven to be an invaluable resource for the ITER tokamak's designers, engineers, and scientists. The lessons learned from the various

\section{J. P. Anderson}

andersonjp@fusion.gat.com

C. Darbos

caroline.darbos@iter.org

1 General Atomics, San Diego, CA 92186-5608PO Box 85608, USA

2 ITER Organization, Route de Vinon sur Verdon, CS 90 046, 13067 St Paul Lez Durance Cedex, France 
experiments discussed at the Workshop and innovative new ideas generated at the Workshop have fed into many of the design decisions being made for ITER. The Workshop has also spurred a large number of extremely useful collaborations. These collaborations have been and continue to be instrumental in advancing various technologies in the area of high-power RF generation, transmission, and radiation.

Last year's Workshop, held in the USA at Sedona, Arizona, in September, was another positive step forward. The participants and organizers all agree that the Workshop was useful, informative, and even fun. The material presented at the Workshop was overall very strong, and therefore it seemed that the time was right to collate the talks into the present Special Issue in the Journal of Infrared, Millimeter, and Terahertz Waves in an effort to document the state of the art in this exciting field.

There are eight reviewed articles in this Special Issue:

1. "Status of the ITER electron cyclotron heating and current drive system" by C. Darbos et al. from ITER Organization in France along with various associates provides an excellent overview of the ECH and current drive system for ITER, from the high voltage power supplies and gyrotrons to the transmission line elements and launchers. The article gives the reader a sense of the major challenges involved when trying to design an industrialgrade system capable of delivering $20 \mathrm{MW}$ of continuous RF power to a plasma at $170 \mathrm{GHz}$.

2. "The multiple gyrotron system on the DIII-D tokamak" by J. Lohr et al. from General Atomics in San Diego, R. Ellis from the Princeton Plasma Physics Laboratory, and L. Riford from Lehigh University, gives an update on significant progress being made on the DIII-D tokamak's ECH system at General Atomics. As reported, the six-gyrotron system is producing 4.7 MW of RF power at $110 \mathrm{GHz}$ for electron cyclotron heating and current drive. An ambitious planned expansion to 10 gyrotrons is also discussed.

3. "Status, operation and extension of the ECRH system at ASDEX Upgrade" by researchers from various institutes in Germany, including D. Wagner from the Max-Planck Institute, as well as T. Goodman from the Centre de Recherches en Physique des Plasmas (CRPP) in Lausanne, Switzerland, A. Litvak et al. from the Institute of Applied Physics in Nizhny Novgorod, and L. Popov et al. from GYCOM in Nizhny Novgorod, discusses recent advances on ASDEX Upgrade's (AUG) ECRH system. The system now uses eight gyrotrons which are used to deliver megawatts of power to the tokamak at $140 \mathrm{GHz}$, and can be tuned to provide power at $105 \mathrm{GHz}$. The launching antennas are able to be controlled real-time during the 10 -s RF pulse to fine-tune the location of power delivery in the tokamak's plasma.

4. "Heating effects in overmoded corrugated waveguide for ITER" by J. Anderson, J. Doane, H. Grunloh, and R. Callis from General Atomics in San Diego, and R. Ikeda, Y. Oda, K. Takahashi, and K. Sakamoto from Japan Atomic Energy Agency in Japan, describes the recent measurement campaign at JAEA's high-power 170-GHz transmission line system, which provides ITER-like conditions. The experiments resulted in some unexpected discoveries, including polarization-dependent heating of waveguide, and led to a new understanding of high-power propagation relevant to ITER.

5. "Mode conversion losses in expansion units for ITER ECH transmission lines" by S. Schaub, M. Shapiro, and R. Temkin from the Massachusetts Institute of Technology, and G. Hanson from US ITER Project in Oak Ridge, TN, describes the development of a 
numerical mode-matching code to predict the propagation losses for expansion waveguide units necessary for ITER's ECH transmission lines.

6. "Development of a propagating millimeter-wave beam position and profile monitor in oversize corrugated waveguide used in an ECRH system" by T. Shimozuma et al. from the National Institute for Fusion Science in Japan provides detail on a new transmission line diagnostic: a real-time monitor of a high-power propagating millimeter-wave beam in an overmoded corrugated waveguide. The device will be able to provide the beam's position and intensity.

7. "Simple expressions for the design of linear tapers in overmoded corrugated waveguides" by S. Schaub, M. Shapiro, and R. Temkin from the Massachusetts Institute of Technology provides straightforward analytical expressions for propagating modes in an overmoded corrugated transmission line undergoing a taper transition to a new diameter. Such tapers are necessary in many ECH transmission lines, including ITERs.

8. "Field formation in the interaction space of gyrotrons" by G. Nusinovich from the University of Maryland at College Park and O. Dumbrajs from the University of Latvia brings a new understanding to the electromagnetic field structures formed in an irregular waveguide typically used as a resonator for high-power gyrotrons. This formulation can be used to help optimize the efficiency of the gyrotron's beam/wave interaction region.

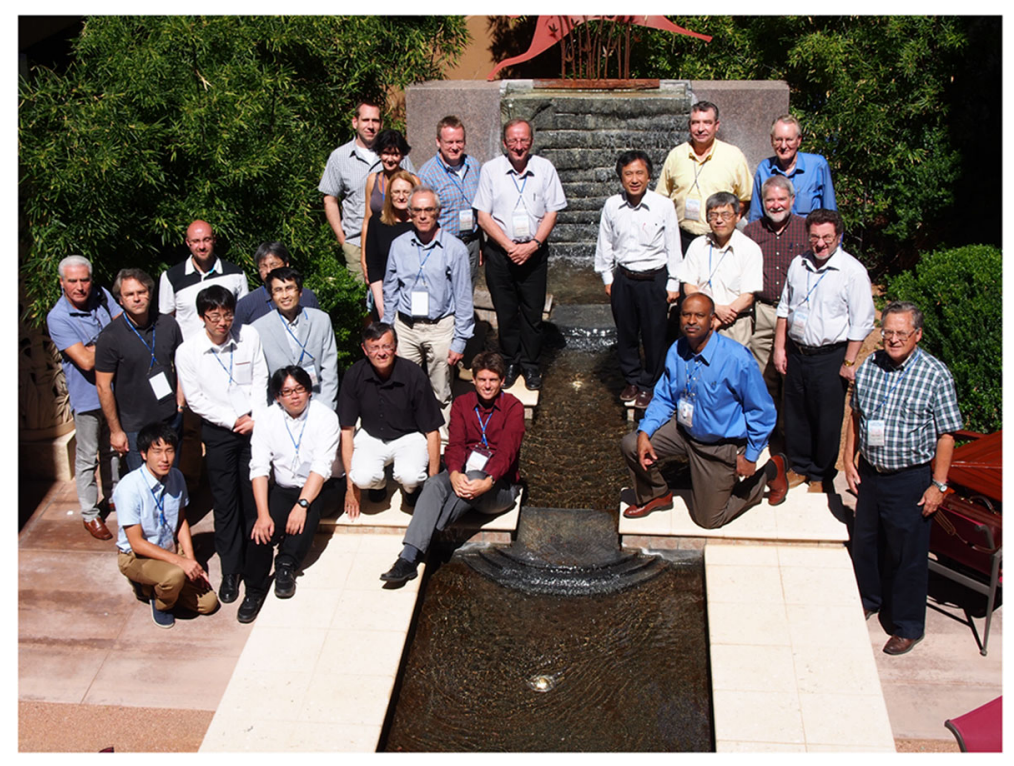

Participants of the 2014 US-EU-JA Workshop on RF Heating Technology of Fusion Plasmas in Sedona, Arizona, US. 